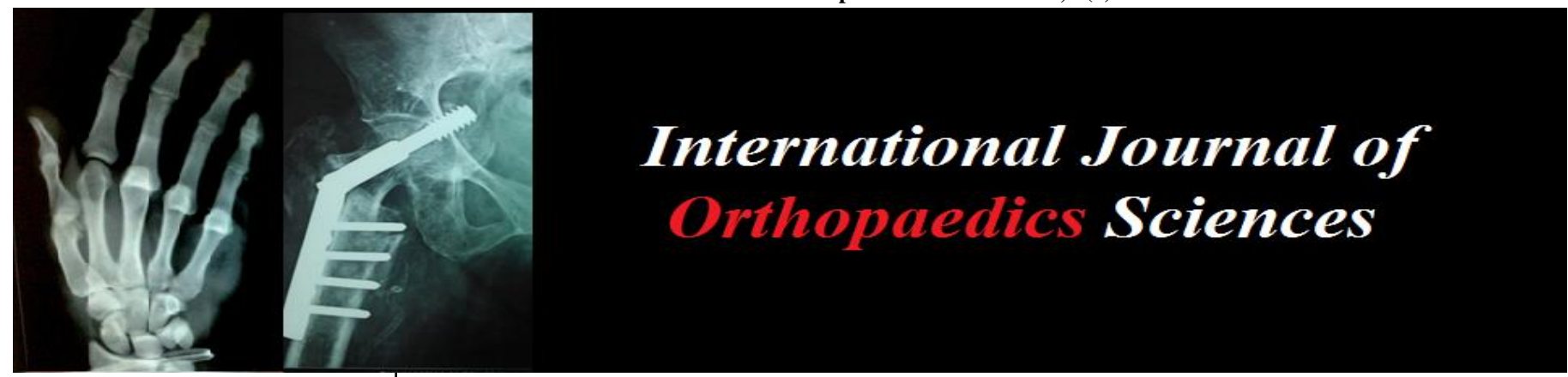

ISSN: $2395-1958$

IJOS 2018; 4(2): 677-681

(C) 2018 IJOS

www.orthopaper.com

Received: 09-02-2018

Accepted: 10-03-2018

Ramesh Perumal

Department of Orthopaedic \& Spine Surgery, Ganga Hospital,

Mettupalayam Road

Coimbatore, Tamil Nadu, India

\section{Sivakumar SP}

Department of Orthopaedic \& Spine Surgery, Ganga Hospital, Mettupalayam Road,

Coimbatore, Tamil Nadu, India

\section{Vijay Shankar}

Department of Orthopaedic \&

Spine Surgery, Ganga Hospital, Mettupalayam Road,

Coimbatore, Tamil Nadu, India

Santosh Ram Gaddam

Department of Orthopaedic \&

Spine Surgery, Ganga Hospital,

Mettupalayam Road,

Coimbatore, Tamil Nadu, India

Dheenadhayalan Jayaramaraju Department of Orthopaedic \& Spine Surgery, Ganga Hospital, Mettupalayam Road,

Coimbatore, Tamil Nadu, India

Shanmuganathan Rajasekaran Department of Orthopaedic \& Spine Surgery, Ganga Hospital, Mettupalayam Road,

Coimbatore, Tamil Nadu, India

Correspondence

Vijay Shankar

Department of Orthopaedic \&

Spine Surgery, Ganga Hospital,

Mettupalayam Road,

Coimbatore, Tamil Nadu, India

\section{Does accelerated bone healing associated with traumatic brain injury (TBI) occur in open fractures}

\section{Ramesh Perumal, Sivakumar SP, Vijay Shankar, Santosh Ram Gaddam, Dheenadhayalan Jayaramaraju and Shanmuganathan Rajasekaran}

DOI: https://doi.org/10.22271/ortho.2018.v4.i2j.99

\section{Abstract}

Aim: Accelerated bone healing has been frequently reported in patients with traumatic brain injury (TBI). However, all these reports are restricted to closed fractures and there is no literature of the effect of TBI in open fractures. Between 2011 and 2013, sixty six patients with TBI of whom thirty four had closed fractures (Group IA) and thirty two had open fractures (Group IIA) were compared with eighty patients without TBI of whom forty had closed fractures (Group IB) and forty had open fractures (Group IIB). The mean duration of follow up was 13 months. Time to fracture union and fracture healing response were compared in both groups. Though group IA patients had shorter time to union (27 weeks) compared to all other groups (IB- 32 weeks, IIA- 36 weeks, IIB-30 weeks), there was no statistical significance between the groups $(P>0.05)$. However, the subset of patients who underwent craniotomy in Group IA (6) had significantly shorter time to healing ( 24 weeks, $\mathrm{P}=0.05$ ) compared to other groups. Considering the fracture healing response (FHR), there was no statistical difference between groups. Accelerated callus response from FHR of 1.4 to 1.8 was observed in closed fractures for thirty six weeks, whereas this was seen only for eighteen weeks in open fractures. In our study of 146 patients, the beneficial effect of TBI on fracture healing was restricted to the subset of patients with closed fractures and those requiring craniotomy. There was no beneficial response in patients with open fractures.

Keywords: Head injury; fracture healing response; craniotomy

\section{Introduction}

Fracture healing response in traumatic brain injury (TBI) has been discussed over few decades and many authors have observed accelerated healing of fractures in $\mathrm{TBI}^{1-4}$. All these studies concentrate only on closed fractures. This study was done to analyze the association of TBI with closed and open fractures of the lower extremity.

\section{Materials and Methods}

Sixty six patients with TBI and lower extremity fractures admitted between January 2011 and December 2013 were included in this study. Among them 34 patients had closed fractures (group IA) and 32 patients had open fractures (group IIA). Patients who had hemorrhagic contusion, hemorrhages (Extradural, Subarachnoid, Intra cerebral) and depressed fractures of the skull were included in the study. Patients without significant computed tomography (CT) findings were excluded. Patients with spinal injuries, history of steroid intake, history of intake of immunosuppressive drugs and patients under the influence of alcohol were excluded from the study. Fractures were classified based on AO classification and for the femur $32 \mathrm{~A}, \mathrm{~B}, \mathrm{C}$ and $33 \mathrm{~A}, \mathrm{~B}, \mathrm{C}$ were included. Tibial fractures which belonged to $42 \mathrm{~A}, \mathrm{~B}$ and $43 \mathrm{~A}$, B were included. Open fractures were classified and managed based on the Ganga Hospital Open Injury Severity Score (GHOISS) proposed by Rajasekaran et al. which specifically addresses the issue of salvage and reconstruction pathways in open injuries ${ }^{[5,6]}$.

In closed fractures with TBI (group IA), there were 34 patients (femur-19 and tibia-15). Group 1A was compared with 40 patients without TBI (group IB) (Table 1). In open fractures with TBI (group IIA) there were 32 patients (femur-4 and tibia-28). They were compared with 40 patients without head injury (group IIB) (Table 2). 
Table 1: Demographic data of closed fractures with TBI Group IA and control group IB

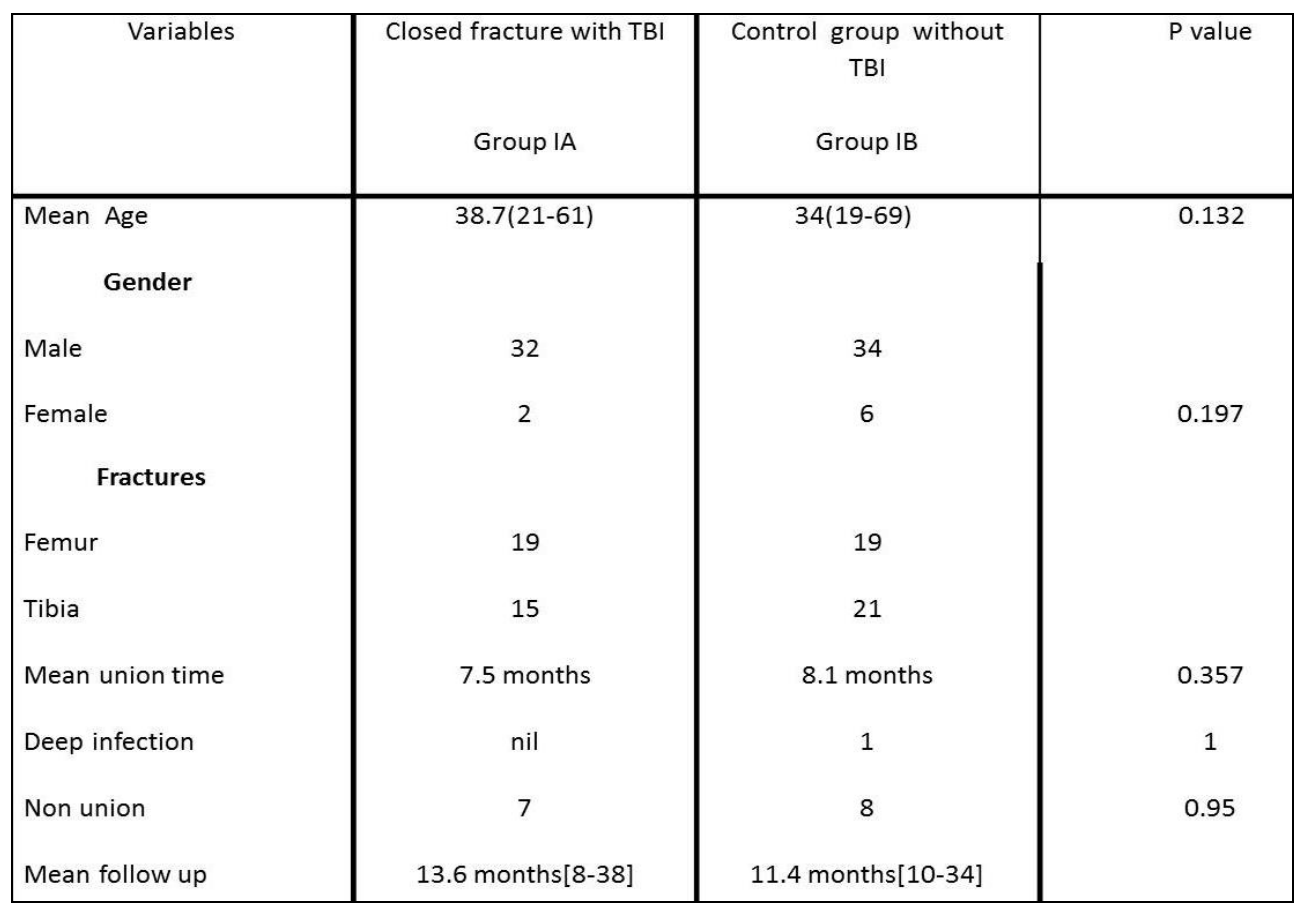

Table 2: Demographic data of open fracture with TBI group IIA and control group IIB

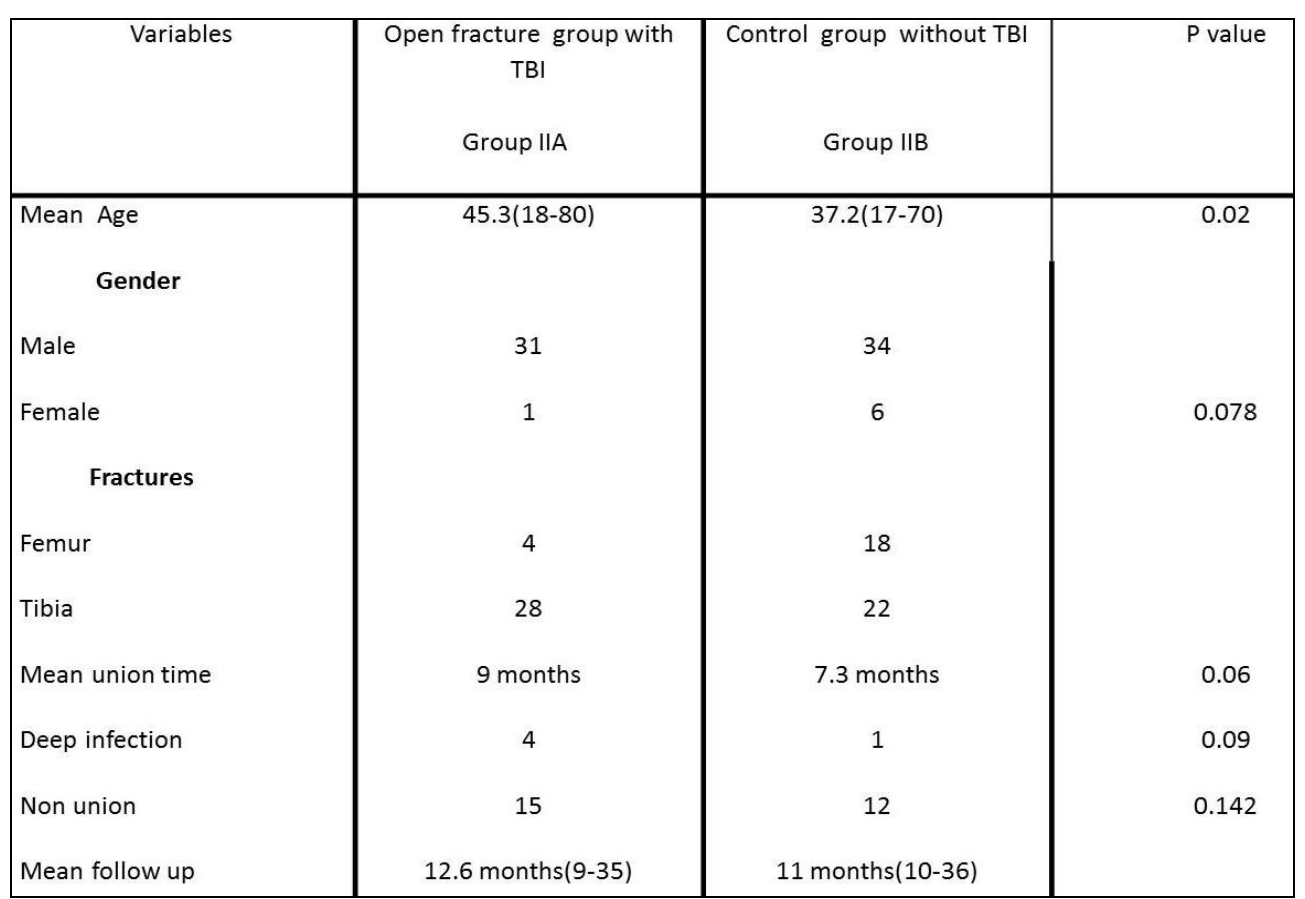

In group IA of 34 patients, 20 underwent primary fixation in the form of intramedullary interlocking nailing and 5 patients underwent definitive external fixation. Remaining 9 underwent initial external fixation followed by nailing. In group IA, ten patients had depressed fractures of the skull, eight had extradural hematoma, eleven had intra-cerebral hemorrhage and five had combinations of subarachnoid hemorrhage, contusions and fractures of the skull. Among them six required a craniotomy for hematoma evacuation and elevation of depressed fractures.

In group IB of 32 patients, twenty two underwent fixation and primary skin closure (interlocking nail-9, plating-4, definitive external fixation-9). Remaining 10 patients underwent initial external fixation followed by nailing. In these ten patients soft tissue cover was done with split skin grafting (2), local flap cover (7) and free tissue transfer (1). In group IB, nine patients had depressed fractures of the skull, five had extradural hematoma, ten had intra-cerebral hemorrhage, five had combinations of subarachnoid hemorrhage, contusions and fractures of the skull, two patients had cerebral infarct and one patient had diffuse cerebral edema. Among them seven required a craniotomy for hematoma evacuation and elevation of depressed skull fracture.

All patients were followed up at regular intervals till union. Fracture healing response (FHR) and time to union were used as outcome measures. FHR was calculated by dividing the diameter of callus by diameter of normal diaphysis in anteroposterior (A) and lateral (B) radiographs ${ }^{[3]}$ (Figure 1). Statistical package for social sciences software for windows version 22.0 was used for statistical analysis. FHR and union time between groups were analyzed using $\mathrm{Z}$ test and ANOVA test. Institutional review board approval was obtained. 


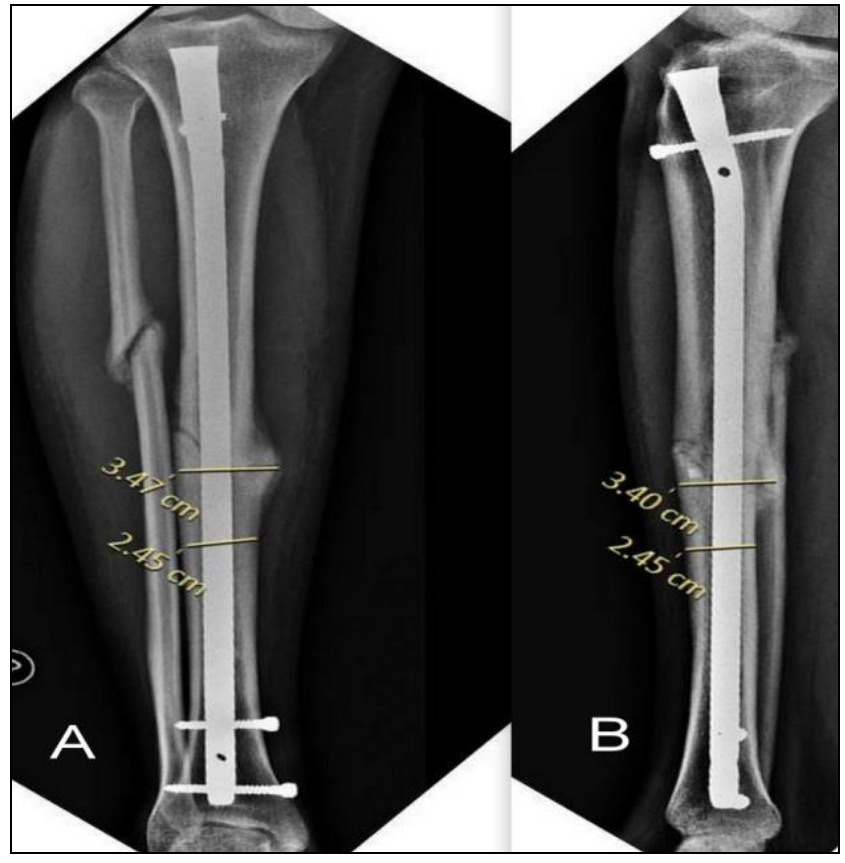

Fig 1: Postoperative radiographs showing callus diameter and normal diaphysis diameter in anteroposterior (A) and lateral (B) radiographs

\section{Results}

In group IA of 34 patients with TBI, there were 32 males and 2 females with mean age of 38.7 years (range: 21-61) which was comparable to the control group IB of 40 patients with the mean age of 34 years (range: 19-69). The mean duration of follow-up was 13.6 months in group IA and 11.4 months in group IB.

The FHR was measured from $6^{\text {th }}$ week onwards serially till 42 weeks follow-up. The FHR in group IA was 1.4 at 6 weeks and gradually increased and reached 1.8 at 36 weeks and followed a downward trend at 42 weeks (Figure 2). However in the control group IB, the FHR hardly reached 1.4 at 30 weeks but most of the times it was around 1.2. Hence there was a significant increase in FHR in group IA (Figure 3). The mean union time was 7.5 months and 8.1 months in group IA and IB respectively. However it was not statistically significant $(\mathrm{P}$ value $=0.352)$. The influence of craniotomy was analyzed in group IA and showed significantly faster union time of 6.1 months (P value=0.05) (Table 3, Figure 4). There were no deep infections in group IA and one deep infection occurred in control group IB. There were 5 non-unions in group IA and 8 non-unions in the control group IB. The parameters of age, gender, deep infection and non-unions in both the groups were not statistically significant.

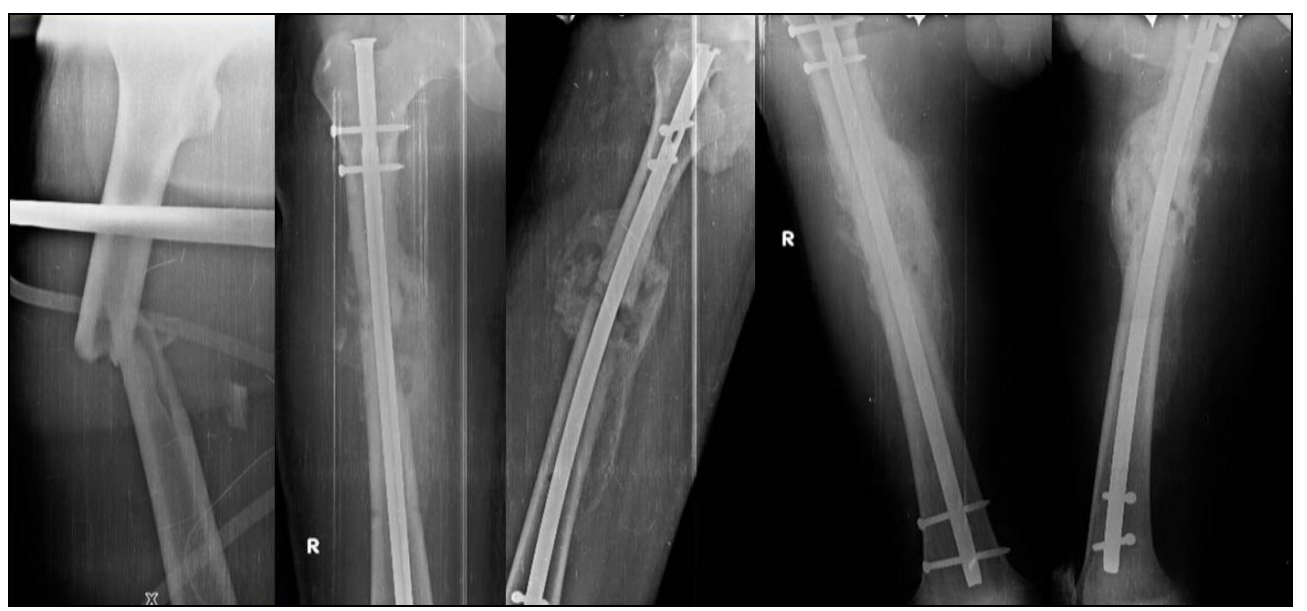

Fig 2: Accelerated callus response in closed femur shaft fracture with TBI treated by interlocking nailing

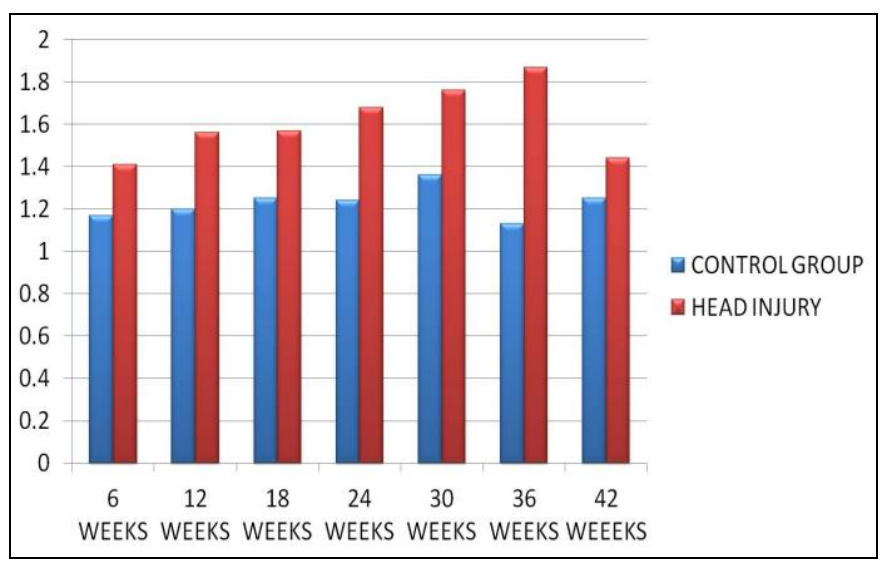

Fig 3: Fracture healing response in group IA and IB
Table 3: Union time comparing patients with and without craniotomy In Group IA

\begin{tabular}{|l|c|c|c|}
\hline \multicolumn{1}{|c|}{ Variables } & \multicolumn{2}{|c|}{ Closed fracture group IA } & Pvalue \\
\hline & No. of patients & $\begin{array}{c}\text { Mean union time[in } \\
\text { months] }\end{array}$ & \\
\hline $\begin{array}{l}\text { Craniotomy done } \\
\text { Craniotomy }\end{array}$ & 6 & 6 & 0.054 \\
$\begin{array}{l}\text { Without head injury } \\
\text { Group IB }\end{array}$ & 32 & 7.3 & \\
\hline
\end{tabular}




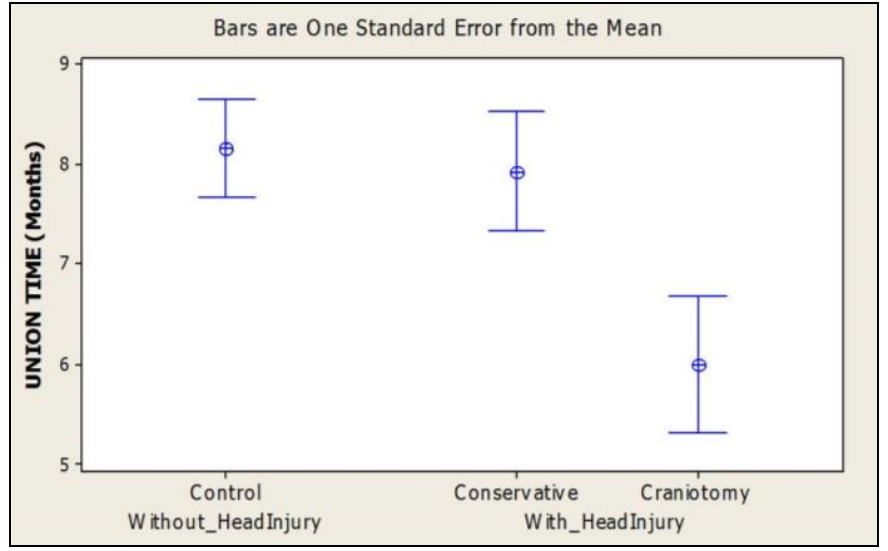

Fig 4: Faster union time in group IA patients who underwent craniotomy

In group IIA of 32 patients with TBI, there were 31 males and 1 female with mean age of 45.3 years which was comparable to the control group IIB of 40 patients with mean age of 37.2 years. The mean duration of follow-up was 12.6 months in group IIA and 11 months in control group IIB.

The FHR in group IIA was 1.4 at 6 weeks and gradually it increased and reached 1.6 at 18 weeks and followed a downward trend later. However in the control group IIB, FHR never reached 1.4 during entire follow-up. Accelerated callus response was observed up to 18 weeks in the head injury group IIA (Figure 5). The mean union time in group IIA was 9 months whereas it was 7.3 months in group IIB. The influence of craniotomy showed no statistical significance in terms of union time $(\mathrm{P}$ value $=0.827)$ (Figure 6 , Table 4$)$. There were 4 deep infections in group IIA and one deep infection in control group IIB. There were 15 non-unions in group IIA and 12 non-unions in control group IIB. There was no statistical difference in terms of age, gender, deep infection and non-union in both the groups.

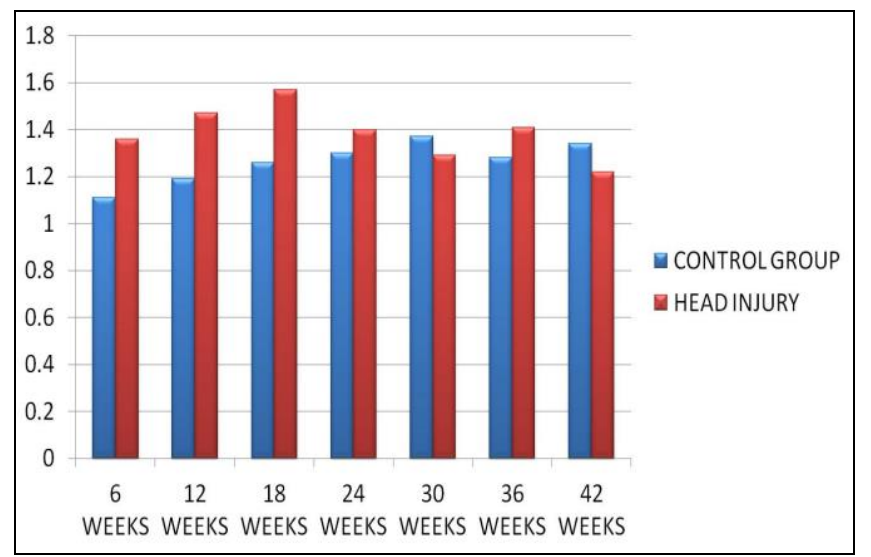

Fig 5: Fracture healing response in group IIA and IIB

Table 4: Union time comparing patients with and without craniotomy In Group IIA

\begin{tabular}{|l|c|c|c|}
\hline \multicolumn{1}{|c|}{ Variables } & \multicolumn{2}{|c|}{ Open fracture group IIA } & P value \\
\hline & No. of patients & $\begin{array}{c}\text { Mean union time[in } \\
\text { months] }\end{array}$ & \\
\hline Craniotomy done & 2 & 10 & 0.827 \\
Without craniotomy & 15 & 8.87 & \\
Without head injury & 28 & 7.3 & 0.06 \\
Group IIB & & & \\
\hline
\end{tabular}

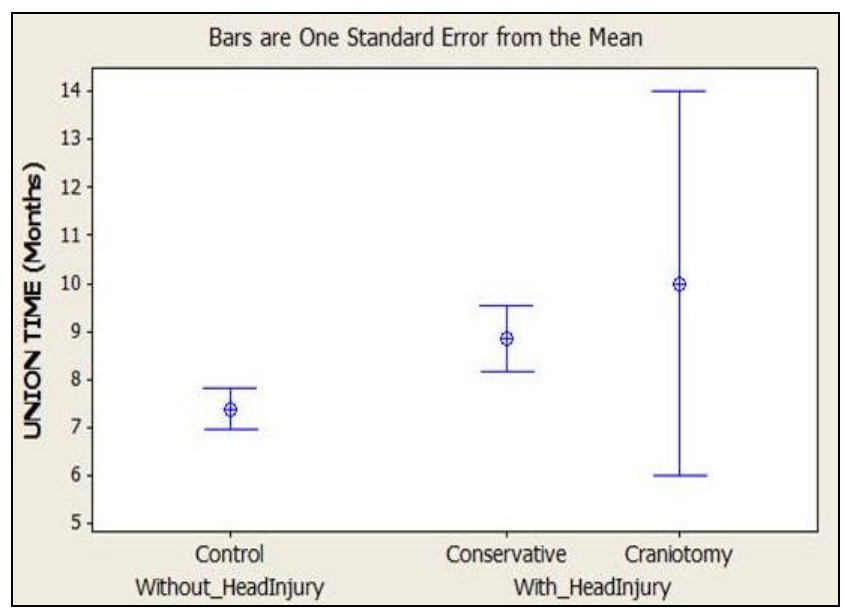

Fig 6: No significant difference in union time in group IIA who underwent craniotomy

\section{Discussion}

The exact nature of the healing response in patients with head injuries has not been fully understood. It is a complex cascade of events which involves local and systemic factors ${ }^{[7-15]}$. This healing response has been described in many terms as "heterotopic ossification" by Garland et al. [16-18], "hypertrophic callus" by Glenn et al. [19], "ossifying hematoma" and "calcifying hematoma" by Keronohan et al. [20]. The studies published in literature so far are limited by small sample size. All these reports are restricted to closed fractures and there is no literature of the effect of TBI in open fractures.

Many authors have shown accelerated fracture healing after TBI ${ }^{[1-3,12-14]}$. Newman et al. reported an increased rate of fracture healing in a small series of 11 patients who had sustained a TBI and an associated long bone fracture, either of the femur or the tibia ${ }^{[1]}$. His findings were comparable to 
results reported by Perkins et al. in a similar group of head injured patients with an associated femoral shaft fracture ${ }^{[2]}$. Our study had the useful addition of a control group with similar femoral fractures. A significant difference was demonstrated in the time to fracture healing between the two groups of patients, with the mean time to union being 89 days in the head-injured group and 110 days in the control group. Spencer et al. ${ }^{[3]}$ reported a group of patients with healing response in long bone fractures including femur, tibia and humerus. He observed that the time to bony union was significantly shorter for fractures of the tibia and humerus, however in femur fractures there was no significant difference. He also analyzed the callus histologically and reported that callus formed at the fracture site in head-injured patients differed significantly from normal callus formation.

A recent study by Yang et al. ${ }^{[4]}$ has also shown that TBI has accelerated fracture healing and enhanced callus formation. A comparative analysis was made between 20 patients with brain injury and 54 without brain injury. He concluded that the severity of injury to brain was not statistically significant in predicting the rate of bone healing and extent of final callus formation.

Garland et al in his study of patients with TBI with diaphyseal tibial, femoral or forearm fractures showed no evidence of increased rate of bone healing. However there were no comparable control groups ${ }^{16-18}$. He also concluded that callus response at the fracture site was a form of heterotopic ossification.

In our study we have compared closed and open fractures with or without TBI. We have observed accelerated healing response in closed fractures with TBI. Another interesting observation was faster union in patients who underwent craniotomy in closed fracture.

In open fractures with TBI, we observed that FHR was accelerated in the early weeks but lesser compared to closed fracture group with TBI. There was also a delay in fracture healing with a mean average union time of 9 months as opposed to 7.3 months in control group. The union time was delayed to 10 months in patients who underwent craniotomy. Local factors like skin healing, tissue loss and lack of adequate hematoma at the fracture site are the main causes of delayed healing in open fractures. However this needs further research with biochemical markers and histological analysis.

\section{Conclusion}

We have observed accelerated healing response in closed fractures with TBI and significantly faster union time in patients who underwent craniotomy. However there was no beneficial response in open fractures with TBI.

\section{Acknowledgments: Nil}

\section{Funding: Nil}

\section{Informed consent: Obtained}

\section{References}

1. Newman RJ, Stone MH, Mukherjee SK. Accelerated fracture union in association with severe head injury. Injury. 1987; 18:241-6.

2. Perkins R, Skirving AP. Callus formation and the rate of healing of femoral fractures in patients with head injuries. J Bone Joint Surg Br. 1987; 69:521-4.

3. Spencer RF. The effect of head injury on fracture healing. A quantitative assessment. J Bone Joint Surg Br. 1987;
69:525-8.

4. Yang TY, Wang TC, Tsai YH, Huang KC. The effects of an injury to the brain on bone healing and callus formation in young adults with fractures of the femoral shaft. J Bone Joint Surg Br. 2012; 94(2):227-30.

5. Rajasekaran S, Sabapathy SR. A philosophy of care of open injuries based on the Ganga hospital score. Injury. 2007; 38:137-46.

6. Rajasekaran S, Naresh Babu J, Dheenadhayalan J, Shetty AP, Sundararajan SR, Kumar M et al. A score for predicting salvage and outcome in Gustilo type-IIIA and

7. Type-IIIB open tibial fractures. The Journal of bone and joint surgery. British. 2006; 88:1351-60.

8. Kadhim HJ, Duchateau J, Sébire G. Cytokines and brain injury: invited review. J Intensive Care Med. 2008; 23:236-249.

9. Rigaux P, Benabid N, Darriet D. Study of serum factors potentially involved in the pathogenesis of heterotopic bone formation after severe brain injury. Joint Bone Spine. 2005; 72:146-149.

10. Cadosch D, Gautschi OP, Thyer M. Humoral factors enhance fracture-healing and callus formation in patients with traumatic brain injury. J Bone Joint Surg [Am]. 2009; 91-A:282-288.

11. Kushwaha VP, Garland DG. Extremity fractures in the patient with a traumatic brain injury. J Am Acad Orthop Surg. 1998; 6:298-307.

12. Roberts P. Heterotopic ossification complicating paralysis of intracranial origin. J Bone Joint Surg Br. 1968; 50-B:70-7.

13. Smith R. Head injury, fracture healing and callus. J Bone Joint Surg Br. 1987; 69:518-20.

14. Jones KB, Mollano AV, Morcuende JA, Cooper RR, Saltzman CL. Bone and brain: a review of neural, hormonal, and musculoskeletal connections. Iowa Orthop J. 2004; 24:123-132.

15. Giannoudis PV, Mushtaq S, Harwood P. Accelerated bone healing and excessive callus formation in patients with femoral shaft fracture and head injury. Injury. 2006; 37:18-24.

16. Morley J, Marsh S, Drakoulakis E, Pape HC, Giannoudis PV. Does traumatic brain injury result in accelerated fracture healing? Injury. 2005; 36:363-368.

17. Garland DE, Dowling V. Forearm fractures in the headinjured adult. Clin Orthop. 1983; 176:190-6.

18. Garland DE, Rothi B, Waters RL. Femoral fractures in head injuries adults. Clin Orthop, 1982, 219-25.

19. Garland DE, Toder L. Fractures of the tibial diaphysis in adults with head injuries. Clin Orthop, 1980, 198-202.

20. Glenn JN, Miner ME, Peitler LF. The treatment of fractures of the femur in patients with head injuries. $\mathbf{J}$ Trauma. 1973; 13:958-61.

21. Kernohan J, Dakin PK, Beacon JP, Bayley JIL. Treatment of major skeletal problems in patients with a severe head injury. Br Med J. 1984; 288:1822-3. 